\title{
Urea C-14
}

National Cancer Institute

\section{Source}

National Cancer Institute. Urea C-14. NCI Thesaurus. Code C47780.

A radiolabelled urea molecule used to diagnose stomach ulcers caused by Heliobacter pylori. In the presence of $\mathrm{H}$. pylori, urea $\mathrm{C}-14$ is metabolized by urease to produce ammonia and radioactive carbon dioxide at the interface between the gastric epithelium and lumen. The radioactive carbon dioxide is absorbed in the blood and is detected when exhaled in the breath. 